\title{
Research Article Circular Microstrip Patch Array Antenna for C-Band Altimeter System
}

\author{
Asghar Keshtkar, ${ }^{1}$ Ahmad Keshtkar, ${ }^{2}$ and A. R. Dastkhosh ${ }^{3}$ \\ ${ }^{1}$ Computer and Electrical Engineering Faculty, Tabriz University, Tabriz, Iran \\ ${ }^{2}$ Medical Physics Department, Medical Faculty, Tabriz University of Medical Sciences, Tabriz, Iran \\ ${ }^{3}$ Electrical Engineering Department, Sahand University of Technology, Tabriz, Iran
}

Correspondence should be addressed to Asghar Keshtkar, akeshtkar@gmail.com

Received 27 February 2007; Accepted 27 November 2007

Recommended by Levent Sevgi

\begin{abstract}
The purpose of this paper is to discuss the practical and experimental results obtained from the design, construction, and test of an array of circular microstrip elements. The aim of this antenna construction was to obtain a gain of $12 \mathrm{~dB}$, an acceptable pattern, and a reasonable value of SWR for altimeter system application. In this paper, the cavity model was applied to analyze the patch and a proper combination of ordinary formulas; HPHFSS software and Microwave Office software were used. The array includes four circular elements with equal sizes and equal spacing and was planed on a substrate. The method of analysis, design, and development of this antenna array is explained completely here. The antenna is simulated and is completely analyzed by commercial HPHFSS software. Microwave Office 2006 software has been used to initially simulate and find the optimum design and results. Comparison between practical results and the results obtained from the simulation shows that we reached our goals by areat degree of validity.
\end{abstract}

Copyright (C) 2008 Asghar Keshtkar et al. This is an open access article distributed under the Creative Commons Attribution License, which permits unrestricted use, distribution, and reproduction in any medium, provided the original work is properly cited.

\section{INTRODUCTION}

Microstrip patch antennas are popular, because they have some advantages due to their conformal and simple planar structure. They allow all the advantages of printed-circuit technology. A vast number of papers are available in the literature, investigating various aspects of microstrip antennas [1-5]. Development of microstrip antennas was initiated in 1981, where a space-borne, light-weight, and low-profile planar array was needed for a satellite communication system. Since then, the development of the microstrip antenna has been expanded into three major program areas: mobile satellite (MSAT) communication, earth remote sensing, and deep-space exploration. The space segment of the MSAT system required the development of an efficient, light-weight, and circularly polarized L-band multiple-beam reflector feed array. In the ground segment, the MSAT required the development of several low-cost and low-profile car-top-mounted L-band antennas. In the area of earth remote sensing, several dual-polarized microstrip arrays are needed for a bistatic radar application, at both the L-band and C-band frequencies, as well as a 1.5-meter-long array at C-band for the aircraft interferometer synthetic aperture radar (SAR) application. In addition, a large Ku-band microstrip planar array (3meter diameter) has been proposed for a scatterometer application.

Finally, a more recent effort calls for the development of a Ka-band MMIC array, as the reflector feed for a future deep-space exploration communication system, as well as a Ka-band array for the advanced communication technology satellite (ACTS) experiment, as a mobile terminal antenna. The design and analysis techniques which have been heavily relied on are the multimode cavity theory and the conventional array theory. Recently, Luk et al. studied the characteristics of the rectangular patch antennas mounted on cylindrical surfaces [6]. Assuming the substrate thickness to be much smaller than wavelength and radius of curvature, they found that the resonant frequencies and the fields under the patch are not affected by curvature. Usually the radiation pattern of a single element is relatively 


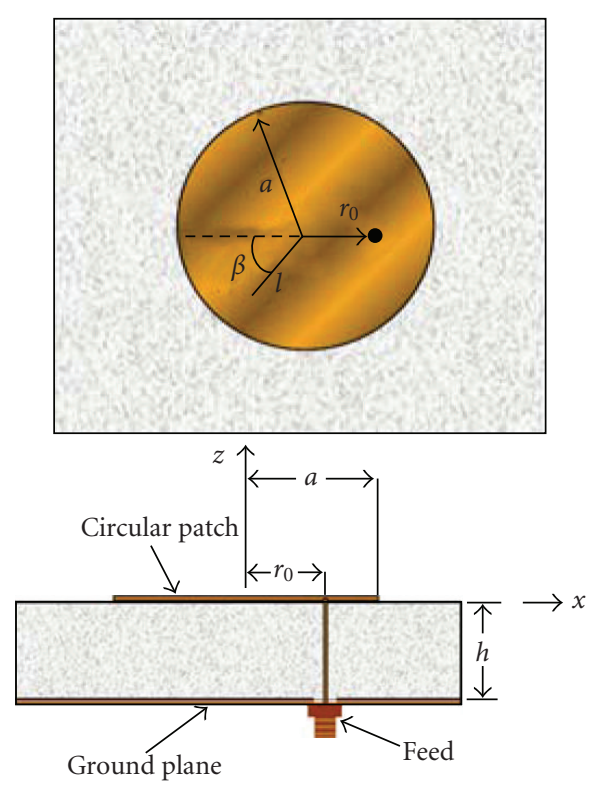

FIGURE 1: Geometry of circular microstrip patch antenna.

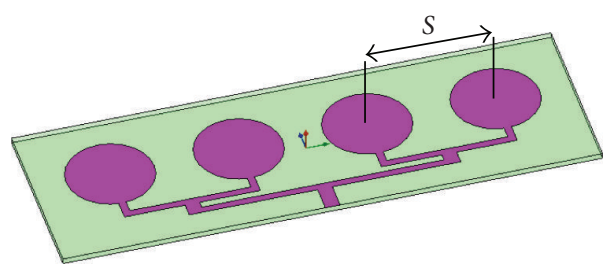

FIGURE 2: Geometry of the array of circular microstrip patch elements.

wide, and each element provides low values of directivity. In many applications, it is necessary to design antennas with very high-directive characteristics to meet the demands of long-distance communications. This can only be accomplished by increasing the electrical size of the antenna. One way to enlarge the dimensions of the antenna without necessarily increasing the size of the individual elements is to form a set of the radiating elements in an electrical and geometrical configuration. This new form of disposing element is designated array. After the rectangular patch, the next most popular configuration is the circular patch or disk.

\section{MATERIALS AND METHODS}

In this paper, an antenna array consisting of four equal circular elements with equal spacing, placed in the $\mathrm{H}$-plane, has been examined (Figures 1 and 2).

In Figure 2, the way of arranging circular patches and feedings is shown (the antenna array is fed from its center). They have the same phase in their entries considering the shapes of feed lines for each of the circular patches.

\subsection{Theory}

In Figure 1, if $h \ll a$ and $h \ll \lambda$, the analysis carried out by Luk et al. [6] showed that, for the rectangular patch, the fields under the cavity are essentially the same as the planar case. It is reasonable to expect that this conclusion is independent of the shape of the patch. For the circular patch, the radial electrical fields of TM modes are given by [6]

$$
E_{\rho}=E_{0} J_{n}\left(k_{n m} l\right) \cos \left[n\left(\beta-\beta_{0}\right)\right],
$$

while $J_{m}(x)$ is the Bessel function of the first kind of order $m$; and $k_{n m}$ is the root of $J_{n}{ }^{\prime}\left(k_{n m} a\right)=0$. Also, " $a$ " that is shown in Figure 1 is the diameter of each of the circular patches. The value of $\beta_{0}$ is determined by the position of the coaxial feed. However, the resonant frequency is

$$
f_{n m}=\frac{k_{n m} c a}{2 \pi a_{e} \sqrt{\varepsilon_{r}}}
$$

where $c$ is the speed of light in free space; and $a_{e}$ is the effective radius that is given by

$$
a_{e}=a\left\{1+\frac{2 h}{\pi a \varepsilon_{r}}\left[\ln \left(\frac{\pi a}{2 h}\right)+1.7726\right]\right\}^{0.5} .
$$

Therefore, the resonant frequency of (2) for the dominant $\mathrm{TM}^{z}{ }_{110}$ should be expressed as

$$
\left(f_{r}\right)_{110}=\frac{1.8412}{2 \pi a_{e} \sqrt{\varepsilon_{r}}} c .
$$

\subsection{Design}

For patch design, it is assumed that the dielectric constant of the substrate $\left(\varepsilon_{r}\right)$, the resonant frequency $\left(f_{r}\right.$ in $\left.\mathrm{Hz}\right)$, and the height of the substrate $h$ (in $\mathrm{cm}$ ) are known.

\section{Design procedure}

A first-order approximation to the solution of (3) for $a$ is to find $a_{e}$ using (4) and to substitute it into (3) for $a_{e}$ and $a$ in the logarithmic function. This will lead to

$$
a=\frac{F}{\left\{1+\left(2 h / \pi \varepsilon_{r} F\right)[\ln (\pi F / 2 h)+1.7726]\right\}^{0.5}},
$$

where

$$
F=\frac{8.791 \times 10^{9}}{f_{r} \sqrt{\varepsilon_{r}}} .
$$

The design of microstrip antenna is done as follows:

$$
f_{r}=4.3 \mathrm{GHz}, \quad h=0.16 \mathrm{~cm}, \quad \varepsilon_{r}=2.33 .
$$

By substituting in (5), $a \mathfrak{J} \leftarrow 1.25 \mathrm{~cm}$.

Richards et al. have reported, calculated, and measured values of the input impedance of a coaxial-feeding rectangular patch with $\varepsilon_{r} \mathfrak{J} \leftarrow 2.62$ and $h \mathfrak{J} \leftarrow 2.62 \mathrm{~cm}$ [7]. For a coaxial feed, matching the antenna impedance to the transmissionline impedance can be accomplished simply by putting the 


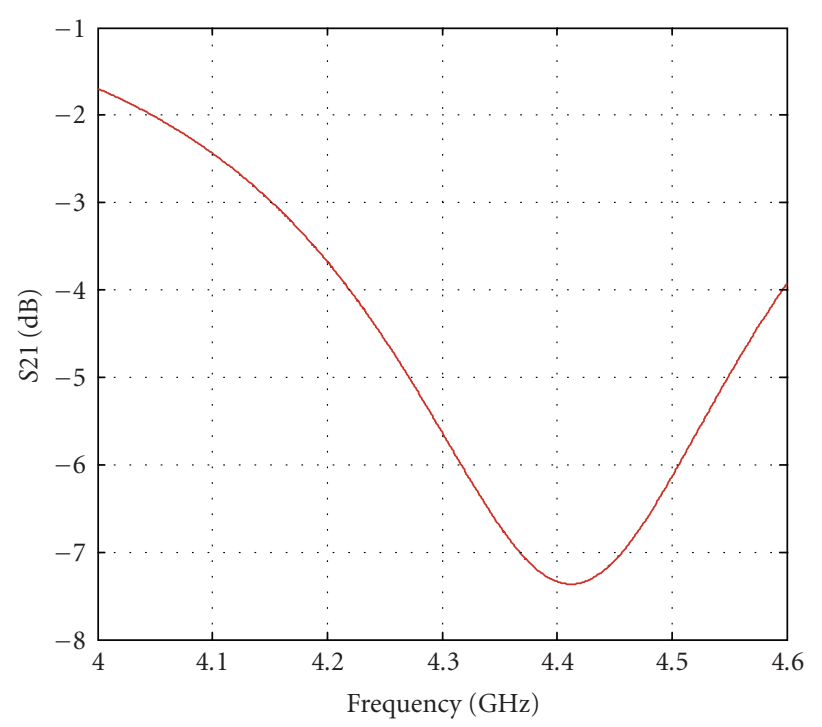

Figure 3: Reflection coefficient as a function of frequency for circular microstrip antenna at $4.3 \mathrm{GHz}$.

feed at the proper location. In [8-11], some formulas have been suggested for computing the input impedance in the resonance state. Typically with very thin substrates, the feed resistance is very smaller than resonance resistance, but in thick substrates, the feed resistance is not negligible and should be considered in impedance matching determining the resonance frequency. In general, the input impedance is complex, and it includes both a resonant part and a nonresonant part which is usually reactive. Both the real and imaginary parts of the impedance vary as a function of frequency. Ideally, both the resistance and reactance exhibit symmetrically about the resonant frequency, and the reactance at resonance is equal to the average of sum of its maximum value (which is positive) and its minimum value (which is negative). A formula that has been suggested to approximate the feed reactance, which does not take into account any images, is [12]

$$
x_{f}=-\frac{\eta k h}{2 \pi}\left[\ln \left(\frac{k d}{4}\right)+0.577\right],
$$

where $d$ is the diameter of the feed probe.

Figure 3 shows the reflection coefficient as a function of frequency simulated with HPHFSS 5.4 software. In the resonance state, the input impedance is a real value and has its maximum quantity. It can be shown that coupling between two patches, as coupling between two apertures or two wire antennas, is a function of the position of one element relative to the other [13-17]. For two circular microstrip patches, the coupling for two side-by-side elements is a function of the relative alignment (Figure 2). In Figure 4, the coupling value that is measured for two cases in E-plane and $\mathrm{H}$-plane is plotted. In this figure, the measure of coupling is plotted versus the distance between centers of two adjacent circular patches.

It can be seen that the coupling in H-plane is very small in comparison with its value in E-plane. It is better to place the elements of the antenna array in $\mathrm{H}$-plane and we showed

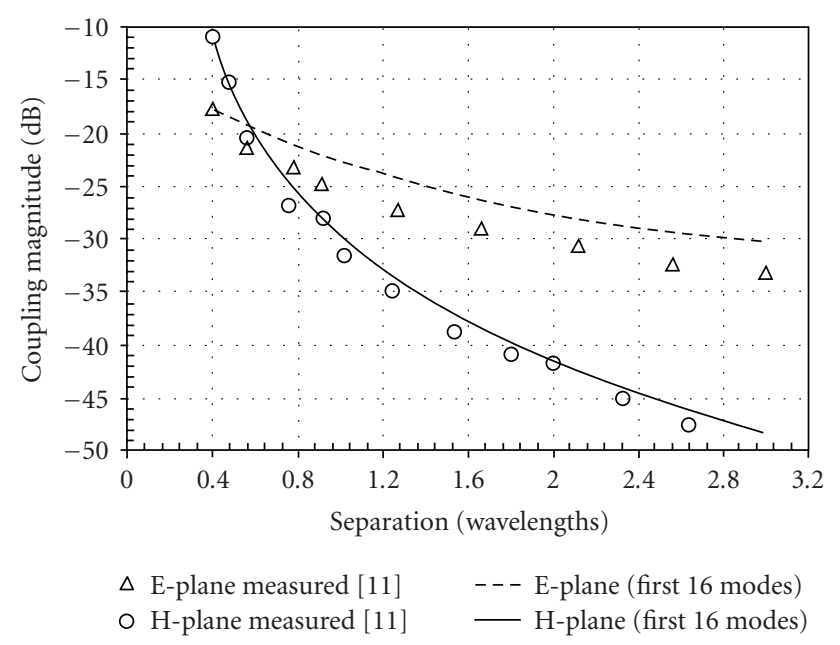

FIgURE 4: Dominant mode mutual coupling for the conventional circular microstrip patch antenna [13].

this in this paper. In the antenna discussed here, the spacing between circular patches $(s)$ is $3.8 \mathrm{~cm}$. Considering that the operating frequency is $4.3 \mathrm{GHz}$, the wavelength will be $7 \mathrm{~cm}$. Consequently, the value of $s / \lambda$ is equal to 1.9 , and then using the plot in Figure 4, the value of coupling is about $-30 \mathrm{~dB}$, that is very small and negligible.

\section{RESULTS AND DISCUSSION}

\subsection{Numerical results and simulation}

The aim of this project is to develop an antenna with a directional pattern and a gain at least equal to $12 \mathrm{~dB}$. An antenna array with equal spacing and uniform excitation was designed. The circular microstrip antenna was simulated by Ansoft Ensemble 8 that is based on the method of moment. For obtaining pattern of this antenna array $(N=4)$, we have the following [18]:

$$
\begin{gathered}
A F=A_{0} \frac{\sin (N \psi / 2)}{N \sin (\psi / 2)}=A_{0} \frac{\sin (2 \psi)}{4 \sin (\psi / 2)}, \\
\psi=\alpha+\beta d \sin (\theta) \cos (\varphi), \\
\beta d=\left(\frac{2 \pi}{\lambda}\right)(s)=\left(\frac{2 \pi}{7}\right)(3.8)=\pi .
\end{gathered}
$$

Here, $\alpha=0$ is the phase difference between elements. Figure 5 shows the array factor. The first null beam width is [18]

$$
\begin{aligned}
& \text { BWFN }=2 \sqrt{\frac{2 \lambda}{N d}}=2 \sqrt{\frac{2 \times 7 \times 10^{-2}}{4 \times 3.8 \times 10^{-2}}}=115^{\circ}, \\
& \mathrm{HPBW}=2 \sqrt{0.886 \frac{\lambda}{N d}}=2 \sqrt{0.886 \frac{7 \times 10^{-2}}{4 \times 3.8 \times 10^{-2}}}=76^{\circ},
\end{aligned}
$$




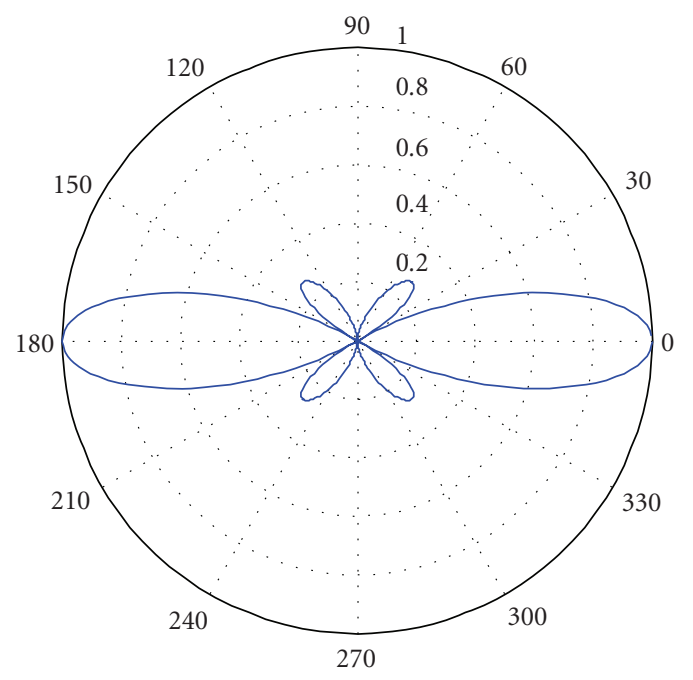

(a)

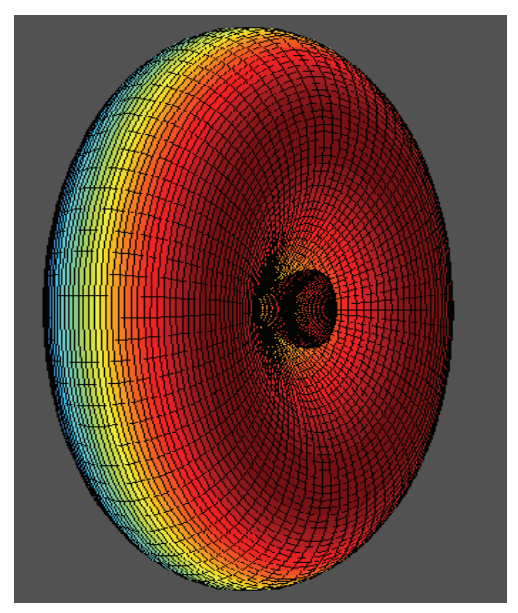

(b)

Figure 5: Array factor for 4 linear elements [18]: (a) H-plane cut and (b) 3D pattern.

where $A F$ in (9) is the array factor and beta $=(2 * \mathrm{pi} / \mathrm{lambda})$, where lambda is the wavelength. "HPBW" is the half-powerbeam width, and "BWFN" is the beam width between first nulls.

These quantities are used to obtain a whole antenna pattern by considering the pattern of a circular microstrip element. The pattern is almost symmetric (in both E-plane and $\mathrm{H}$-plane), and side lobes are very small (Figure 6). A plot of the directivity of the dominant $\mathrm{TM}^{z}{ }_{110}$ mode as a function of the radius of the disk is shown in Figure 7 . The measurement of antenna parameters by theoretical calculations is somehow difficult, but we can calculate them easily by softwares, such as HPHFSS. In general, the dependence of antenna parameters to their physical parameters can be mentioned. The bandwidth is inversely proportional to $\sqrt{\varepsilon_{r}}$. As we know, the total directivity is equal to multiplication of patch directivity and to the array directivity. From the diagrams in Figures 5 and 7 , the value of directivity factor of circular microstrip antenna is about $5 \mathrm{~dB}$, and then a directivity of $13 \mathrm{~dB}$ at $4.3 \mathrm{GHz}$

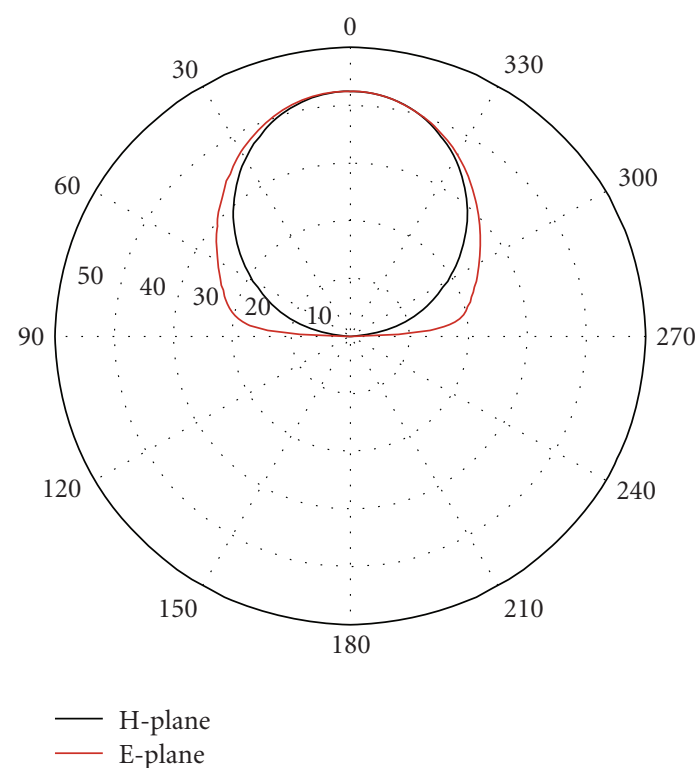

FIGURE 6: Computed (based on moment method and cavity models) E-plane and H-plane patterns of circular microstrip patch antenna: E-plane $\left(\phi=0^{\circ}, 180^{\circ}\right)$, H-plane $\left(\phi=90^{\circ}, 270^{\circ}\right)$.

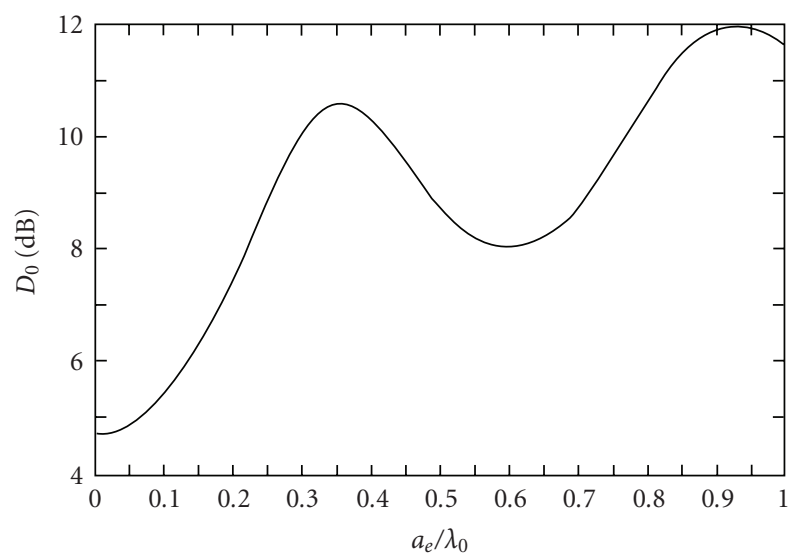

FIGURE 7: Directivity versus effective radius for circular microstrip patch antenna operating in dominant $\mathrm{TM}_{110}^{z}$ mode [12].

is achieved with the main lobe in the broadside direction, with the 50-degree HPBW, and $25 \mathrm{~dB}$ SLL below the main lobe.

The circular microstrip patch array antenna was simulated by Ansoft Ensemble 8 and is shown in Figure 6.

In contribution to our discussion, we consider the results of the simulation. In the analysis of this antenna by HPHFSS software, three-dimensional pattern of this antenna is obtained. It is shown in Figure 8, and its pattern in E-plane and $\mathrm{H}$-plane is also shown in Figure 9.

Considering the obtained value of input impedance by HPHFSS software, we can obtain the matching impedance by Microwave Office software (or analytic methods). Considering the feed lines, the circular patches have the same phase at their entries, as can be seen in Figure 2, we specify the impedance in each line by varying the width of lines until 


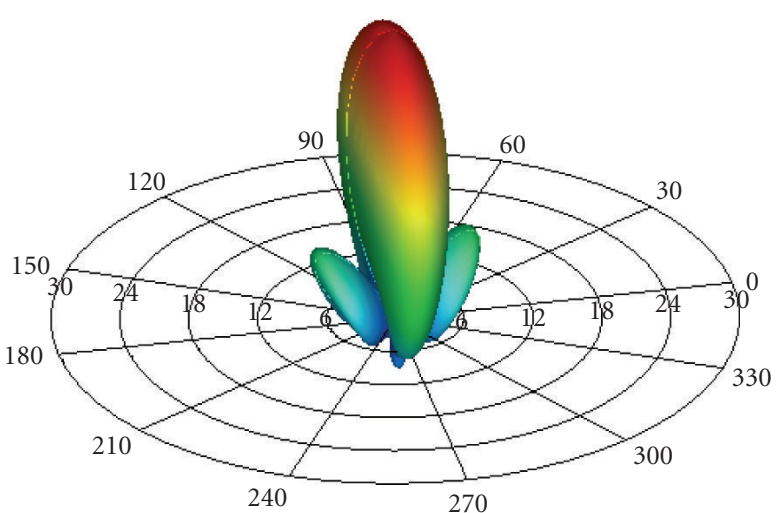

(a)

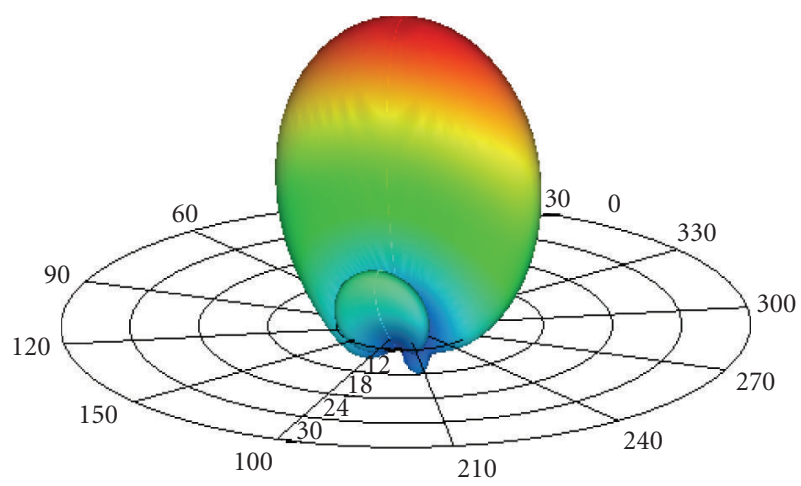

(b)

FIGURE 8: Three-dimensional pattern of the array antenna with the circular microstrip patches simulated with the HPHFSS 5.4: (a) $\phi=$ $0^{\circ}$, (b) $\phi=90^{\circ}$.

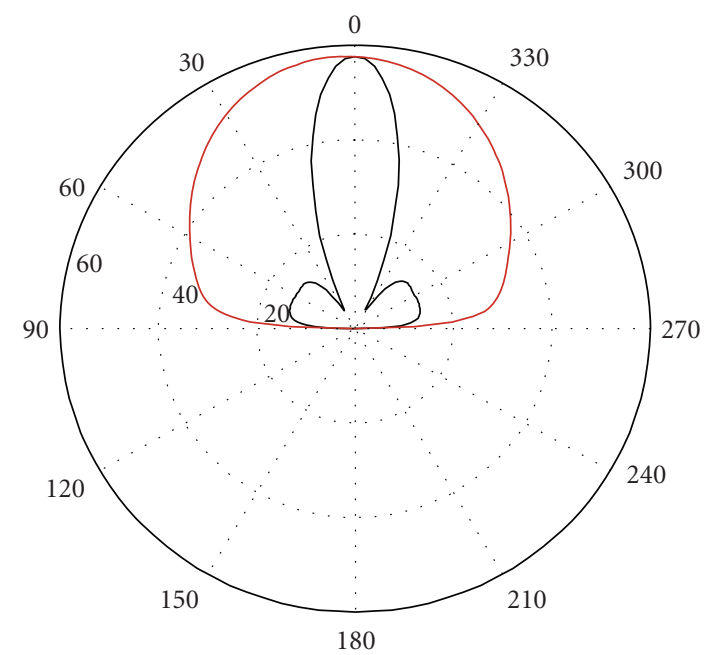

- H-plane

- E-plane

FIgURE 9: E-plane and H-plane patterns of the array antenna with the circular microstrip patches simulated with the HPHFSS 5.4.

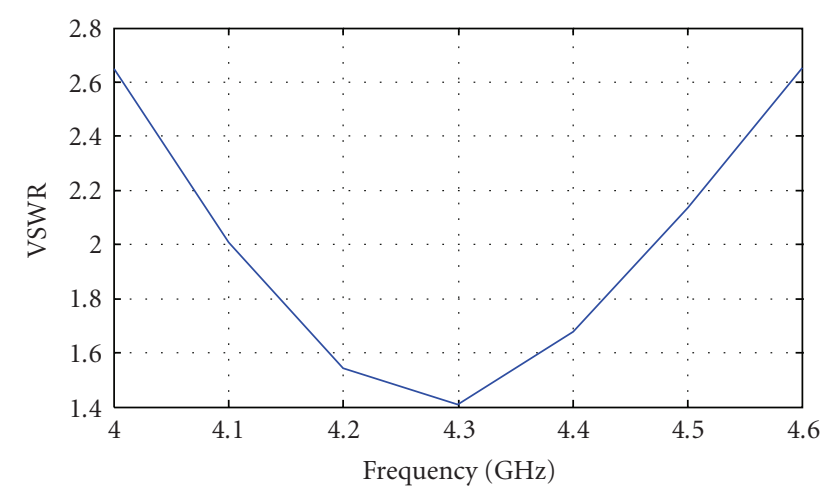

FIgURE 10: VSWR as a function of frequency simulated by Microwave Office.

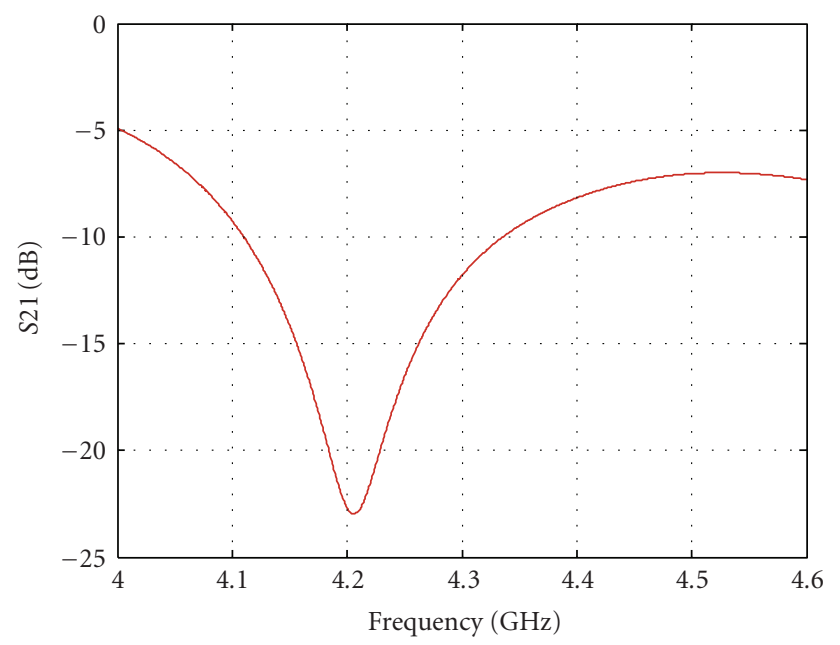

FIGURE 11: Reflection coefficient versus frequency of the array antenna with the circular microstrip patches simulated with the HPHFSS 5.4.

we obtain the amount of input impedance equal to $50 \Omega$. The value of VSWR for this antenna that is obtained by Microwave Office software is shown in Figure 10, and the value of reflection coefficient that is obtained by HPHFSS software is shown in Figure 11. Regarding the simulation with HPHFSS 5.4 software, the input impedance of the antenna is $55+25 j$, and so we have

$$
\begin{gathered}
|\Gamma|=\left|\frac{Z_{l}-Z_{0}}{Z_{l}+Z_{0}}\right|=0.2362=-12.5 \mathrm{~dB}, \\
\operatorname{VSWR}=\frac{1+|\Gamma|}{1-|\Gamma|}=1.6 .
\end{gathered}
$$

\subsection{Array antenna configuration}

The array antenna was constructed as shown in Figure 12. The dimensions and structural diagram of the antenna are shown too in this figure. The fabricated patch was designed to operate at $4.3 \mathrm{GHz}$. The patch is probe feeding, and the ground plane is finite for this patch and has the dimensions of $15 \stackrel{m}{=} \leftarrow 4 \mathrm{~cm}$. By selecting proper values for microstrip-line width and length and the position of the feed point, a good 


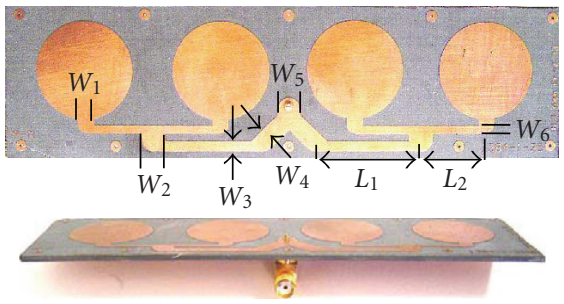

FIGURE 12: Pictures of the fabricated antenna and its geometry. The feeding line is a standard $50 \Omega$ coaxial probe feed. Other dimensions are $W_{1}=0.4 \mathrm{~cm}, W_{2}=0.6 \mathrm{~cm}, W_{3}=0.3 \mathrm{~cm}, W_{4}=0.5 \mathrm{~cm}, W_{5}=$ $0.6 \mathrm{~cm}, W_{6}=0.2 \mathrm{~cm}, L_{1}=3 \mathrm{~cm}, L_{2}=1.9 \mathrm{~cm}$.

Mstp ant. $01 \quad$ H-plane \& E-plane pattern \#Date 83/10/22

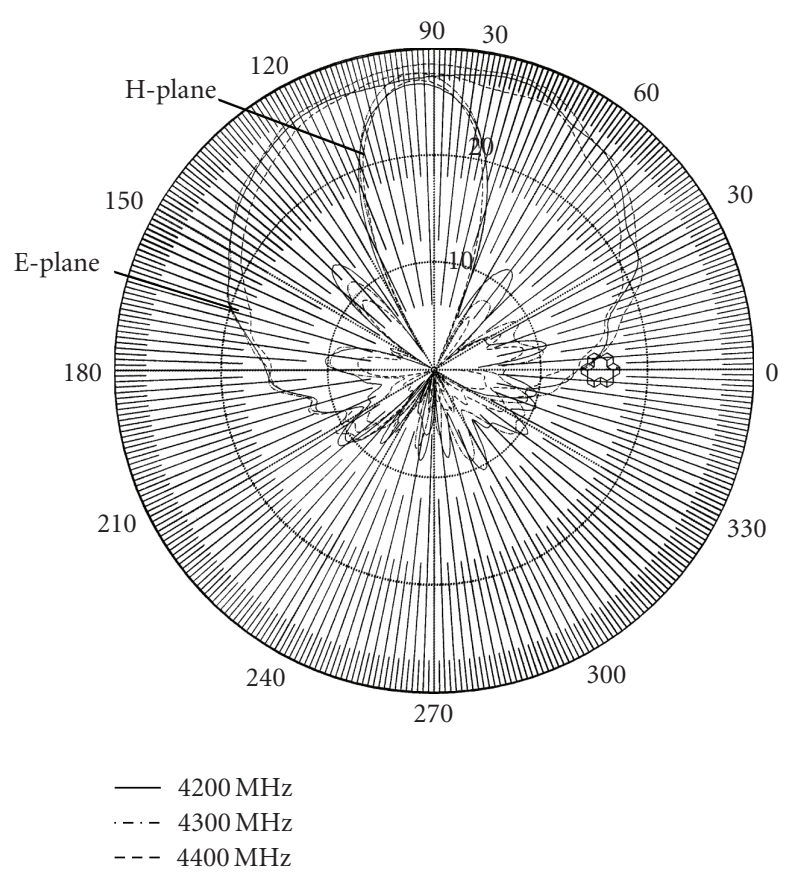

K.N Toosi Univ. of Tech. Telecom. Dept. Prof. Morshed Ant. Lab.

FIgURE 13: Measured E-plane and H-plane patterns of the array antenna with the circular microstrip patches.

impedance bandwidth can be obtained. An inset feed scheme is employed to match the patch antenna to a $50 \Omega$ coaxial probe feed. The dielectric material has a permittivity of 2.33 and a thickness of $0.16 \mathrm{~cm}$. The substrate of this antenna is made of RT/Duroid 5870, fabricated by Rogers Company (Mentor, OH, USA).

\subsection{Results of the test}

In the test process, the antenna pattern and the value of VSWR were obtained. Antenna radiation performance was measured and recorded in two orthogonal principal planes (E-plane and $\mathrm{H}$-plane or vertical and horizontal planes). The pattern was plotted in the form of polar coordinates. By definition, near-field tests are done by sampling the field very

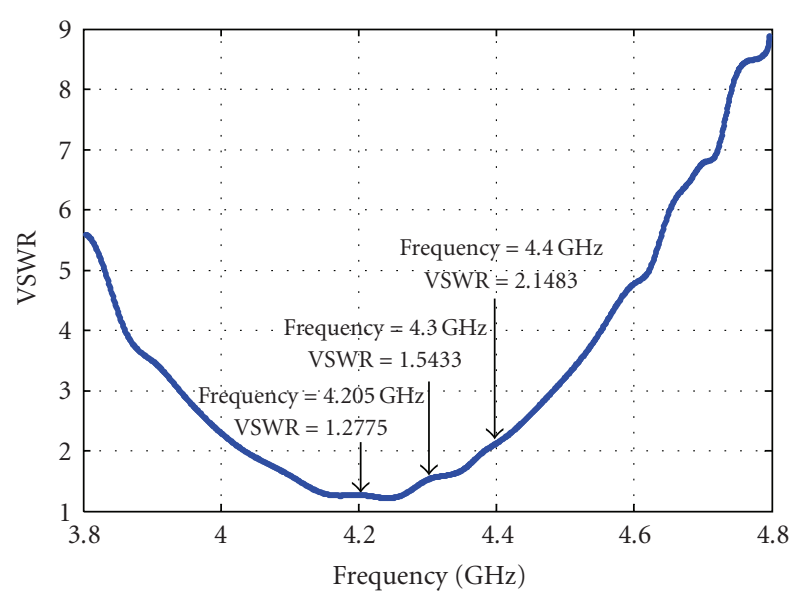

FIGURE 14: VSWR as a function of frequency measured by the AGILENT 8510C network analyzer.

close to the antenna on a known surface. From the phase and amplitude data collections, the far-field pattern was computed in the same fashion that theoretical patterns were computed from the theoretical field distributions. The transformation used in the computation depends on the shape of the surface over which the measurements are taken with the scanning probe. An antenna range instrumentation must be designed to operate over a wide range of frequencies, and it usually can be classified into five categories as follows:

(1) source antenna and transmitting system,

(2) receiving system,

(3) positioning system,

(4) recording system,

(5) data-processing system.

This technique involves an antenna under test which is placed on a rotational positioned and rotated around the azimuth to generate a two-dimensional polar pattern. This measurement was done for the two principal axes of the antenna to determine parameters such as antenna beam width in both the E- and H-planes. The practical results of the test are in agreement with the desirable results and theoretical analysis. E-plane and H-plane patterns of the antenna are shown in Figure 13. In the practical test carried out by AGILENT 8510C network analyzer, the value of VSWR in central frequency was 1.5433 that was well in agreement with the theoretical analysis (Figure 14). Variation in the measured performance is mainly due to imprecise fabrication by a milling machine. It is important to calibrate the network analyzer before doing VSWR measurement. The network analyzer should be calibrated for a suitable frequency range containing the band where the antenna will operate. Typical network analyzers have a cable with SMA connector in the end. Calibration was performed by connecting three known terminations, $50 \Omega$ load, short, and open, to this SMA connector. After calibration the reference plane will be at the connection point of the SMA connector. To measure the reflection at the feed point of the antenna, a semirigid coax cable with SMA connector in one end can be used. 


\section{CONCLUSION}

A small microstrip patch antenna array has been presented. The antenna has been designed to be used in altimeter system applications, in the C-band. In fact, this antenna was designed for $4.3 \mathrm{GHz}$ and $12 \mathrm{~dB}$ gain. But as you can see, 4.2 GHz also has good pattern and proper VSWR. The design has been accomplished using commercially available HPHFSS, Ansoft Ensemble 8, and Microwave Office 2006 softwares. The designed antenna has shown good performance in terms of return losses and radiation (a prototype has been fabricated and tested). Good agreement has been obtained between simulation and experimental results, providing validation of the design procedure. Good performance has been obtained for the envisaged applications.

\section{REFERENCES}

[1] J.-S. Kuo and K.-L. Wong, "A compact microstrip antenna with meandering slots in the ground plane," Microwave and Optical Technology Letters, vol. 29, no. 2, pp. 95-97, 2001.

[2] J.-S. Kuo and K.-L. Wong, "Dual-frequency operation of a planar inverted-L antenna with tapered patch width," Microwave and Optical Technology Letters, vol. 28, no. 2, pp. 126-127, 2001.

[3] F. Ferrero, C. Luxey, G. Jacquemod, and R. Staraj, "Dual-band circularly polarized microstrip antenna for satellite applications," IEEE Antennas and Wireless Propagation Letters, vol. 4, no. 1, pp. 13-15, 2005.

[4] J. E. C. Neto, G. F. B. Oliveira, and H. C. C. Fernandes, "Analysis of planar antenna array," in Proceedings of the SBMO/IEEE MTT-S International Microwave and Optoelectronics Conference (IMOC '03), vol. 1, pp. 323-325, Iguaza Falls, Brazil, September 2003.

[5] N. Crispim, R. Peneda, and C. Peixeiro, "Small dual-band microstrip patch antenna array for MIMO system applications," in Proceedings of IEEE Antennas and Propagation Society International Symposium (APS '04), vol. 1, pp. 237-240, Monterey, Calif, USA, June 2004.

[6] K.-M. Luk, K.-F. Lee, and J. S. Dahele, "Analysis of the cylindrical-rectangular patch antenna," IEEE Transactions on Antennas and Propagation, vol. 37, no. 2, pp. 143-147, 1989.

[7] W. Richards, Y. T. Lo, and D. D. Harrison, "An improved theory for microstrip antennas and applications," IEEE Transactions on Antennas and Propagation, vol. 29, no. 1, pp. 38-46, 1981.

[8] D. M. Pozar, "Microstrip antennas," Proceedings of the IEEE, vol. 80, no. 1, pp. 79-91, 1992.

[9] R. Garg, P. Bhartia, I. Bahl, and I. B. A. Ittiboon, Microstrip Antenna Design Handbook, Artech House, London, UK, 2001.

[10] T. A. Milligan, Modern Antenna Design, John Wiley \& Sons, New York, NY, USA, 2nd edition, 2005.

[11] K.-L. Wong, Compact and Broadband Microstrip Antennas, Wiley-InterScience, New York, NY, USA, 2002.

[12] C. A. Balanis, Antenna Theory, Analysis and Design, John Wiley \& Sons, New York, NY, USA, 2nd edition, 1997.

[13] M. A. Khayat, J. T. Williams, D. R. Jackson, and S. A. Long, "Mutual coupling between reduced surface-wave microstrip antennas," IEEE Transations on Antenna and Propagation, vol. 48, no. 10, pp. 1581-1593, 2000.

[14] H. T. Hui, "Reducing the mutual coupling effect in adaptive nulling using a re-defined mutual impedance," IEEE Mi- crowave and Wireless Components Letters, vol. 12, no. 5, pp. 178-180, 2002.

[15] T. Su and H. Ling, "On modeling mutual coupling in antenna arrays using the coupling matrix," Microwave and Optical Technology Letters, vol. 28, no. 4, pp. 231-237, 2001.

[16] B. Belentepe, "Modeling and design of electromagnetically coupled microstrip-patch antennas and antenna arrays," IEEE Antennas and Propagation Magazine, vol. 37, no. 1, pp. 31-39, 1995.

[17] A. J. Sangster and R. T. Jacobs, "Mutual coupling in conformal microstrip patch antenna arrays," IEE Proceedings: Microwaves, Antennas and Propagation, vol. 150, no. 4, pp. 191-196, 2003.

[18] W. L. Stutzman and G. A. Thiele, Antenna Theory and Design, John Wiley \& Sons, New York, NY, USA, 2nd edition, 1981. 

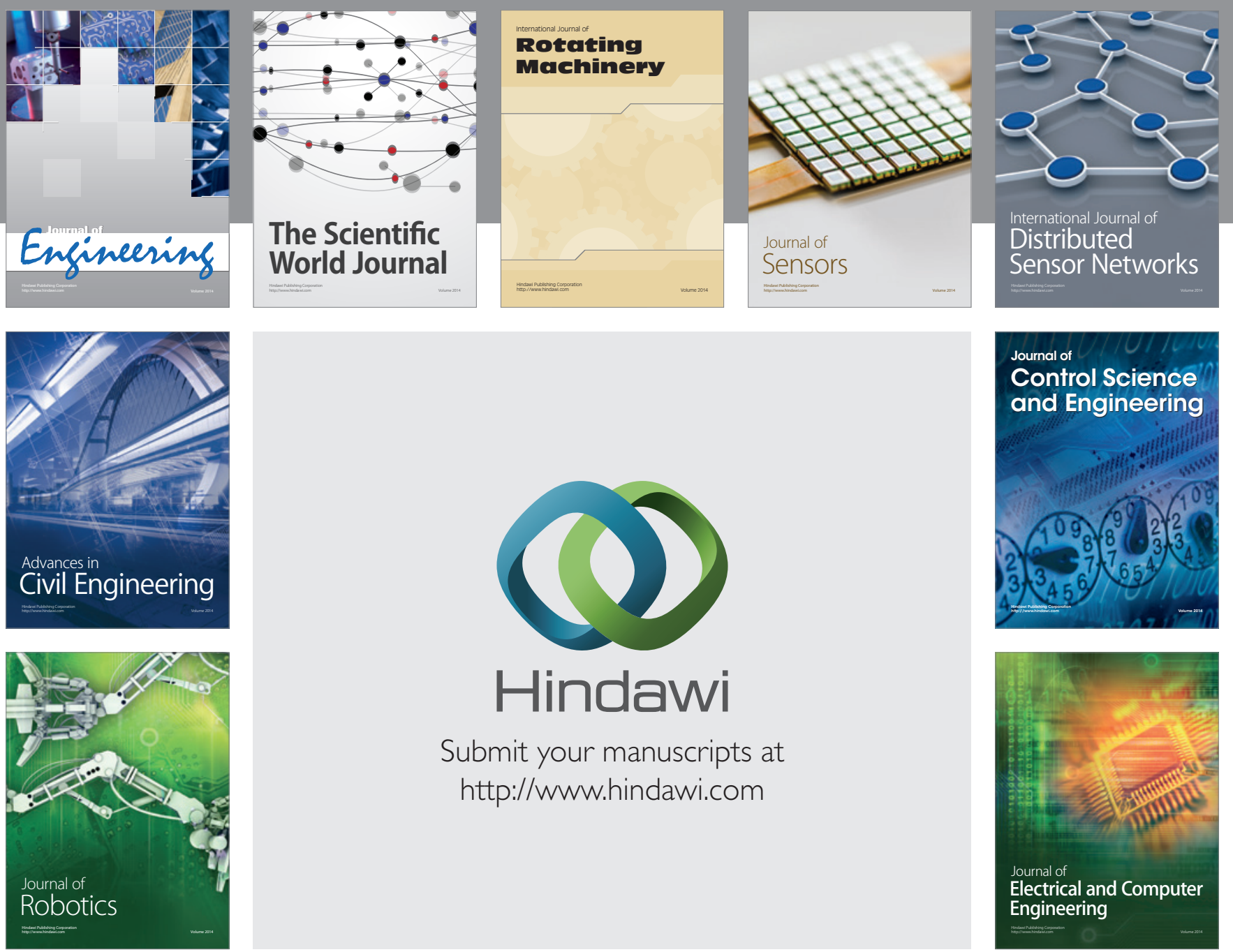

Submit your manuscripts at

http://www.hindawi.com
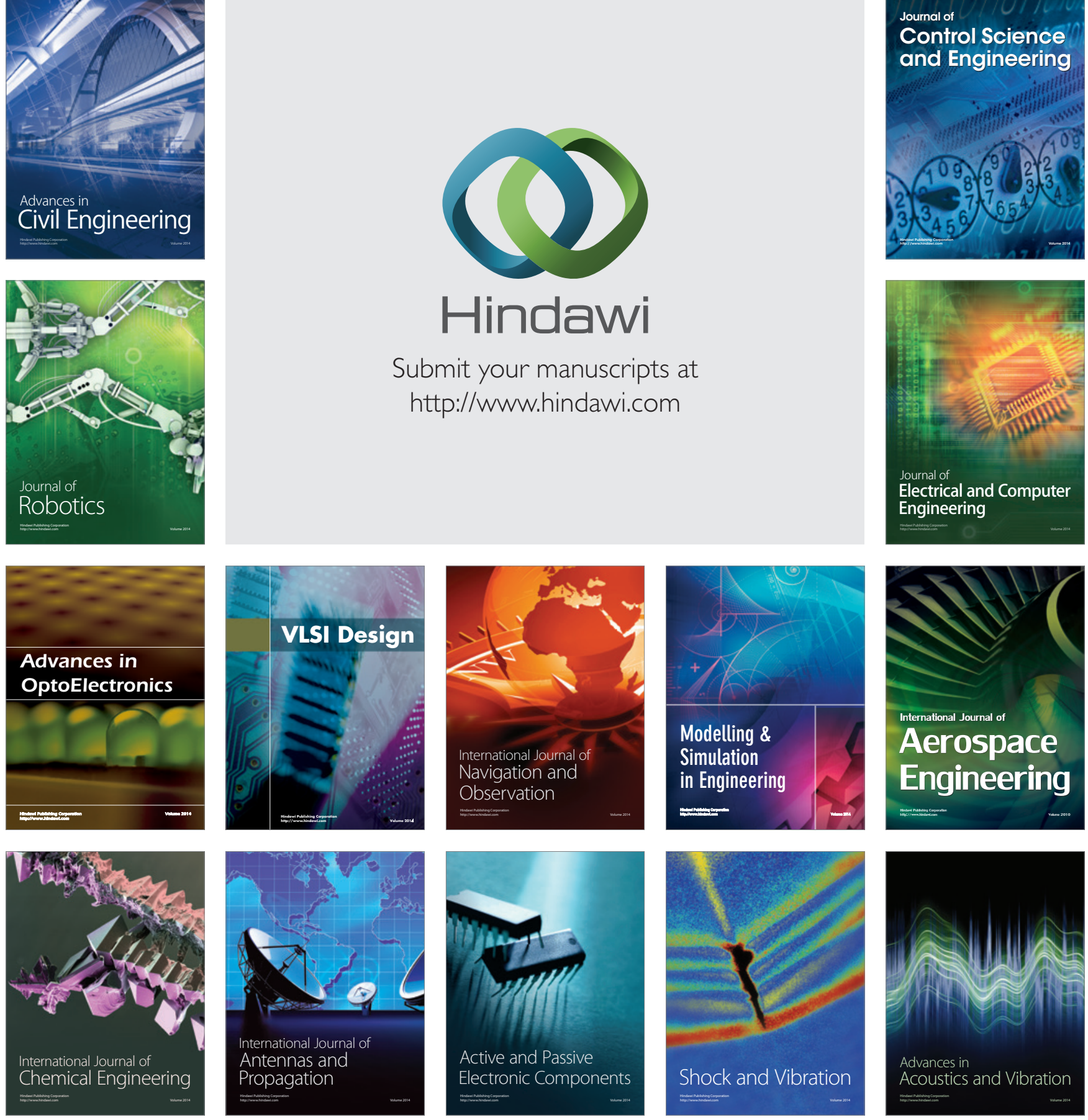